\title{
P01-10-4 Poster session
}

\section{Factors modifying the length of stay in the Medical Intensive Care Unit of a tertiary care teaching hospital in India}

\author{
Manish Bajpai, Sartaj Hussain, Suraj Yadav, Sanjay Khattri, Kamal K Sawlani \\ Physiology, King George's Medical University, UP, Lucknow, India
}

Background: Longer length of stay (LOS) in the intensive care unit is associated with increases the risk of nosocomial infections, mortality and high costs compared to shorter LOS.

Methods: A prospectively observational study was conducted in the Intensive Care Unit (ICU) of Medicine department of a tertiary care teaching hospital in North India. Patients who stayed for less than 24 hours were excluded from study. The LOS is divided into two parts: shorter (\&\#8804;5 days) and longer ( $>5$ days). A multiple regression binary logistic with forward conditional elimination was used to identify independent predictors of length of stay \& outcome.

Results: A total of 101 patients were recruited. The mean LOS (95\% Confidence Interval) was 7.11 days (5.70-8.52) and range of 1-50 days. The mortality was significantly higher in the patient with longer LOS ( $>5$ days). On univariate analysis, the significant factors for length of stay were Acute Physiology and Chronic Health Evaluation (APACHE) II score at admission, ventilation and nosocomial infection. The multivariate analysis showed that the independent predictor for length of stay was gender, APACHE II Score at admission, comorbidity, ventilation and nosocomial infection.

Conclusions: The predictors for prolonged intensive care unit stay were gender, APACHE II Score at admission, comorbidity, ventilation and nosocomial infection. 